\title{
Genomic analysis of xCT-mediated regulatory network: identification of novel targets against AIDS-associated Iymphoma
}

\author{
Lu Dai ${ }^{1,3}$, Yueyu Cao${ }^{1}$, Yihan Chen ${ }^{1}$, Johnan A.R. Kaleeba ${ }^{5}$, Jovanny Zabaleta ${ }^{4}$ and \\ Zhiqiang Qin ${ }^{1,2}$ \\ ${ }^{1}$ Research Center for Translational Medicine and Key Laboratory of Arrhythmias of the Ministry of Education of China, East \\ Hospital, Tongji University School of Medicine, Shanghai, China \\ 2 Department of Microbiology/Immunology/Parasitology, Louisiana State University Health Sciences Center, Louisiana Cancer \\ Research Center, New Orleans, LA, USA \\ ${ }^{3}$ Department of Medicine, Louisiana State University Health Sciences Center, Louisiana Cancer Research Center, New \\ Orleans, LA, USA \\ ${ }^{4}$ Department of Pediatrics, Louisiana State University Health Sciences Center, Louisiana Cancer Research Center, New \\ Orleans, LA, USA \\ ${ }^{5}$ Department of Microbiology and Immunology, Uniformed Services University of the Health Sciences, Bethesda, MD, USA \\ Correspondence to: Zhiqiang Qin, email: zqin@Isuhsc.edu
}

Keywords: KSHV, herpesvirus, XCT, lymphoma, microarray

Received: January 29, $2015 \quad$ Accepted: March 10, $2015 \quad$ Published: March 30, 2015

This is an open-access article distributed under the terms of the Creative Commons Attribution License, which permits unrestricted use, distribution, and reproduction in any medium, provided the original author and source are credited.

\section{ABSTRACT}

Kaposi's sarcoma-associated herpesvirus (KSHV) is the etiological agent of primary effusion lymphoma (PEL), a rapidly progressing malignancy mostly arising in HIV-infected patients. Even under conventional chemotherapy, PEL continues to portend nearly $100 \%$ mortality within several months, which urgently requires novel therapeutic strategies. We have previously demonstrated that targeting $\mathrm{XCT}$, an amino acid transporter for cystine/glutamate exchange, induces significant PEL cell apoptosis through regulation of multiple host and viral factors. More importantly, one of XCT selective inhibitors, Sulfasalazine (SASP), effectively prevents PEL tumor progression in an immune-deficient xenograft model. In the current study, we use Illumina microarray to explore the profile of genes altered by SASP treatment within $3 \mathrm{KSHV}^{+}$ PEL cell-lines, and discover that many genes involved in oxidative stress/antioxidant defense system, apoptosis/anti-apoptosis/cell death, and cellular response to unfolded proteins/topologically incorrect proteins are potentially regulated by XCT. We further validate 2 downstream candidates, OSGIN1 (oxidative stress-induced growth inhibitor 1 ) and XRCC5 (X-ray repair cross-complementing protein 5 ), and evaluate their functional relationship with PEL cell survival/proliferation and chemoresistance, respectively. Together, our data indicate that targeting these novel XCT-regulated downstream genes may represent a promising new therapeutic strategy against PEL and/or other AIDS-related lymphoma.

\section{INTRODUCTION}

The oncogenic Kaposi's sarcoma-associated herpesvirus (KSHV, also known as Human herpesvirus 8) is a principal causative agent of several human cancers including primary effusion lymphoma (PEL), which arises preponderantly in immunocompromised individuals, in particular acquired immune deficiency syndrome (AIDS) patients [1]. PEL usually comprises transformed B cells harboring KSHV episomes and presents as pleural, peritoneal and pericardial neoplastic effusions [2]. PEL is a rapidly progressing malignancy with a median survival time of approximately 6 months even under conventional chemotherapy [3]. Furthermore, the myelosuppressive effects of systemic cytotoxic chemotherapy synergize with those caused by antiretroviral therapy or immune 
suppression [2-4], which supports the need to identify novel targets that could guide development of more effective therapeutic strategies. Recently, we found that the amino acid transporter, $\mathrm{xCT}$, is highly expressed on the surface of patient-derived $\mathrm{KSHV}^{+} \mathrm{PEL}$ cells, and targeting $\mathrm{xCT}$ by pharmacological inhibition or RNAi silencing induces significant PEL cell apoptosis [5]. We further demonstrated that the underlying mechanisms for this effect include regulation of both host and viral factors: (i) reduction of intracellular glutathione (GSH) synthesis and increased reactive oxygen species (ROS) production, (ii) repression of cell-proliferation-related signaling, in particular Akt pathway, and (iii) induction of viral lytic gene expression [5]. More importantly, we demonstrated that an XCT selective inhibitor, Sulfasalazine (SASP), effectively prevents PEL tumor progression in an immune-deficient xenograft model [5], which suggests that $\mathrm{xCT}$ may represent a promising target for AIDSrelated lymphomas.

The expression of $\mathrm{xCT}$ on the cell membrane is essential for the uptake of cystine required for synthesis of intracellular glutathione (GSH), an anti-oxidant that plays an important role in maintaining the intracellular redox balance $[6,7]$. Therefore, $\mathrm{xCT}$ is highly expressed by a variety of malignant tumors because the uptake of cystine/cysteine from the microenvironment is crucial for cancer cell growth and viability [8-11]. Interestingly, $\mathrm{xCT}$ has also been identified as a fusion-entry receptor for $\mathrm{KSHV}[12,13]$, which is upregulated within more advanced Kaposi's sarcoma (KS, another KSHV-related malignancy[14]) lesions containing a greater number of KSHV-infected cells [15]. We also recently reported that $\mathrm{xCT}$ is able to activate intracellular signaling pathways (in particular MAPK), pro-migratory cytokine release, and $\mathrm{KSHV}$-infected endothelial cell invasion through induction of the $14-3-3 \beta$ protein [16]. Moreover, $\mathrm{xCT}$ is functionally involved in the pathogenesis of other viruses and bacteria as well [17-20]. However, the mechanisms of xCT-mediated regulation of KSHV pathogenesis and tumorigenesis, including the $\mathrm{xCT}$ regulatory network in AIDS-related lymphomas such as $\mathrm{KSHV}^{+} \mathrm{PEL}$ remain unknown. Clearly, innovative insights from this information would facilitate the identification of potential "drug target" candidates for development of new therapeutic strategies. Therefore, in the current study we used Illumina human microarray analysis to interrogate changes in the transcriptional profiles of genes in $3 \mathrm{KSHV}^{+}$PEL cell-lines treated with the $\mathrm{xCT}$ selective inhibitor, SASP, which led to identification of a number of novel xCT-regulated downstream genes important to PEL survival or chemoresistance.

\section{RESULTS}

\section{Microarray analysis of xCT-regulated network within $\mathrm{KSHV}^{+}$PEL cells}

We first used the HumanHT-12 v4 Expression BeadChip (Illumina) which contains more than 47,000 probes derived from the NCBI RefSeq Release 38 and other sources to study the gene profile altered between vehicle- or SASP-treated $3 \mathrm{KSHV}^{+}$PEL cell-lines (BCP$1, \mathrm{BC}-1$ and $\mathrm{BCBL}-1)$. Intersection analysis indicated that there were totally 100 common genes significantly altered within all the 3 SASP-treated cell-lines (up/ down $\geq 2$ folds and $p<0.05$ ); 33 similar genes altered between BCBL-1 and BC-1, 93 similar between BCBL1 and $\mathrm{BCP}-1$, and 124 similar between $\mathrm{BC}-1$ and $\mathrm{BCP}-$ 1; 80 genes altered were unique to $\mathrm{BCBL}-1,150$ unique to $\mathrm{BCP}-1$ and 640 unique to $\mathrm{BC}-1$ (Figure 1). Notably, $\mathrm{BC}-1$ cells, which are also $\mathrm{EBV}^{+}$, had a much higher number of uniquely altered genes than BCBL-1 and BCP1. Within the common gene set, the top 20 upregulated or downregulated candidate genes in SASP-treated BCP-1, BC-1 and BCBL-1 cell-lines are listed in Table 1 and Table 2, respectively, including gene description and the altered level of transcription in these cell-lines. Interestingly, we found that the functional role of most genes in PEL pathogenesis have never been reported, although some of them have been implicated in other types of malignancies. For example, SRXN1 (Sulfiredoxin-1), which is upregulated in all three SASP-treated PEL cell lines (Table 1) is involved in proliferation inhibition of acute myeloid leukemia mediated by Maesopsin 4-O-betaD-glucoside, a natural compound isolated from the leaves of Artocarpus tonkinensis) [21]. Another study reported that activation of PFKP (6-phosphofructokinase type C), which is downregulated in SASP-treated PEL cells (Table 2 ) is closely associated with breast cancer cell proliferation [22]. The opposite effects of SASP on SRXN1 and PFKP transcription underscores the putative benefits of this drug in clinical management of PEL as well. We next performed enrichment analysis of these common, similar and unique sets of genes using the Pathway map, Gene Ontology (GO) Processes and Process Networks modules from Metacore Software (Thompson Reuters) [23]. Our analysis shows that several major cellular functions were affected within SASP-treated PEL cells, including oxidative stress/ antioxidant defense system, apoptosis/anti-apoptosis/ cell death, and cellular response to unfolded proteins/ topologically incorrect proteins, which is consistent with the SASP-induced apoptosis phenotype that we recently observed in $\mathrm{KSHV}^{+} \mathrm{PEL}$ cell-lines [5]. The top 2 scored pathway maps and protein networks based on the enrichment analysis of "common" gene set were listed in Figures 3 and S1, respectively. 
Table 1: The top 20 candidate genes upregulated in KSHV+ PEL cells treated by SASP.

\begin{tabular}{|l|c|c|c|c|}
\hline \multirow{2}{*}{ Gene symbol } & Description & \multicolumn{3}{c|}{ Fold change } \\
\cline { 3 - 5 } & OSCP-1 & BC-1 & BCBL-1 \\
\hline OSGIN1 & Oxidative stress-induced growth inhibitor 1 & 14.73 & 5.85 & 7.87 \\
\hline HSPA6 & Heat shock 70 kDa protein 6 & 4.36 & 14.97 & 4.75 \\
\hline MSC & Musculin & 7.51 & 8.53 & 5.46 \\
\hline DHRS2 & Dehydrogenase/reductase SDR family member 2, mitochondrial & 10.64 & 5.75 & 4.36 \\
\hline PPP1R15A & Protein phosphatase 1 regulatory subunit 15A & 6.77 & 6.14 & 6.45 \\
\hline HSPA1A & Heat shock 70 kDa protein 1 & 7.05 & 5.57 & 5.24 \\
\hline SRXN1 & Sulfiredoxin-1 & 7.53 & 3.03 & 5.85 \\
\hline GCLM & Glutamate--cysteine ligase regulatory subunit & 6.24 & 4.12 & 5.25 \\
\hline SQSTM1 & Sequestosome-1 & 7.46 & 2.24 & 5.3 \\
\hline RN7SK & RNA, 7SK small nuclear transcript & 4.98 & 4.62 & 4.81 \\
\hline HSPA7 & Putative heat shock 70 kDa protein 7 & 3.04 & 7.69 & 3.44 \\
\hline DNAJB1 & DnaJ homolog subfamily B member 1 & 5.81 & 4.67 & 3.48 \\
\hline DNAJC3 & DnaJ homolog subfamily C member 3 & 3.87 & 7.39 & 2.46 \\
\hline LILRB3 & Leukocyte immunoglobulin-like receptor subfamily B member 3 & 4.71 & 3.4 & 4.59 \\
\hline RNF141 & RING finger protein 141 & 5.23 & 3.3 & 3.86 \\
\hline C6orf52 & DNA finger CW-type PWWP domain protein 1 cell-surface antigen heavy chain & 3 & 5.69 & 2.42 \\
\hline CCL3L3 & Putative uncharacterized protein C6orf52 & 4.16 & 4.63 & 2.28 \\
\hline ZCWPW1 & C-C motif chemokine 3-like 1 & 3.51 & 2.97 & 4.33 \\
\hline SLC3A2 & DNage-inducible transcript 3 protein & 4.66 & 4.11 & 2.43 \\
\hline DDIT3 & 2 & 3.17 \\
\hline
\end{tabular}

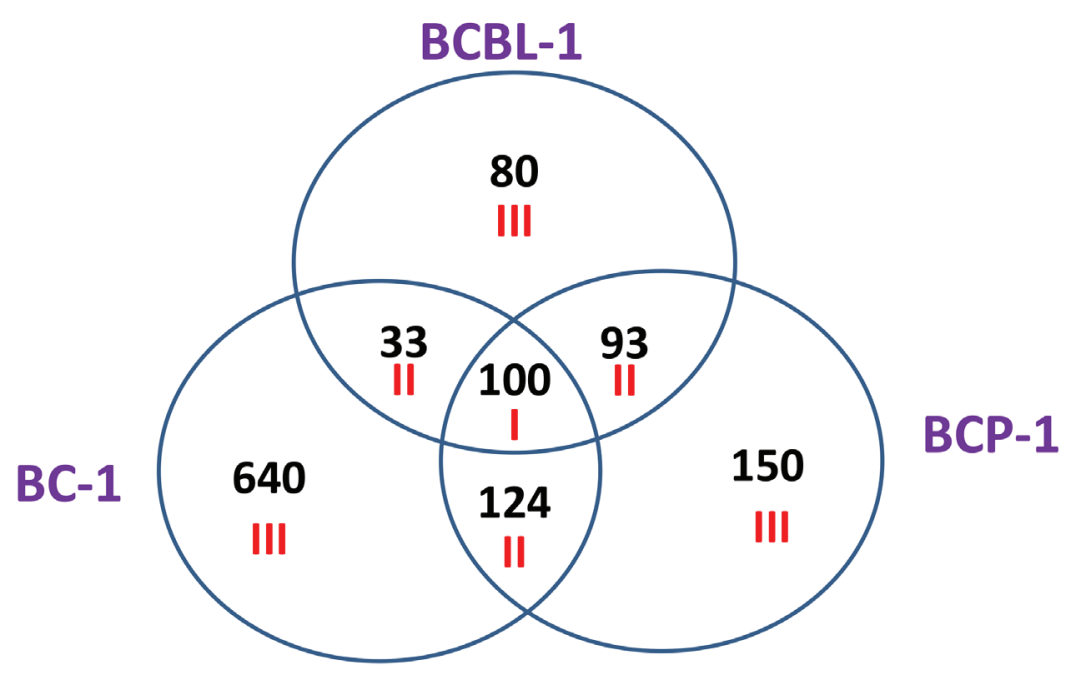

Figure 1: Intersection analysis of gene profile altered within SASP-treated PEL cell-lines. The HumanHT-12 v4 Expression BeadChip (Illumina) was used to detect genomic gene profile altered within 3 SASP-treated PEL cell-lines (BCBL-1, BC-1 and BCP-1) when compared with vehicle-treated controls. Intersection analysis of significantly altered genes (up/down $\geq 2$ folds and $p<0.05$ ) was performed using the Illumina GenomeStudio Software. Set I: Common genes altered in all the 3 cell-lines; Set II: Similar genes altered in every 2 cell-lines; Set III: Unique genes altered in each cell-line. 


\section{Experimental validation of microarray results with selected downstream candidates}

We next selected 5 genes from the top 20 upregulated or downregulated candidate list (Tables 1 and 2) for validation of their transcriptional change by qRT-PCR. Our results indicated that all the 5 genes (OSGIN1, HSPA6, DHRS2, PPP1R15A and HSPA1A) were significantly upregulated in SASP-treated PEL cells when compared with vehicle-treated cells; while another 5 genes (ASZ1, ARL4C, NREP, LGALS13, PPIA) were all significantly downregulated in SASP-treated PEL cells (Figure 4). Moreover, the altered transcriptional levels of these genes in all the $3 \mathrm{KSHV}^{+}$PEL cell-lines (BCBL$1, \mathrm{BC}-1$ and $\mathrm{BCP}-1)$ were comparable to those found in microarray data, demonstrating the credibility of our microarray analysis.

\section{The role of OSGIN1 in SASP-induced $\mathrm{KSHV}^{+}$ PEL cell apoptosis}

We next selected OSGIN1 (Oxidative stress-induced growth inhibitor 1), one of the highly upregulated genes in SASP-treated $\mathrm{KSHV}^{+} \mathrm{PEL}$ cells from microarray data, to determine its role in SASP-induced cell apoptosis. The OSGIN1 gene (also named as OKL38) was first identified in breast epithelial cells as increasingly expressed during pregnancy and lactation [24]. Low-level expression of this gene has been reported in breast cancer cell lines, while its overexpression in MCF-7 breast cancer cells leads to a reduction in proliferation as well as tumor formation in nude mice [24]. As a tumor suppressor, downregulation of OSGIN1 was also found to be closely associated with progression of other malignancies such as hepatocellular carcinoma and kidney cancer [2527]. Here, we found that silencing of OSGINI by RNAi significantly reduced cell apoptosis induced by SASP $(0.5 \mathrm{mM})$ in BCP-1 and BCBL-1 cells (Figures $5 \mathrm{~A}$ and $\mathrm{S} 2$ ). Western blot analysis also indicated that silencing of OSGIN1 by RNAi in SASP-treated BCP-1 and BCBL-1 greatly reduced cleaved Caspase 3 and 9, while partially rescuing the phosphorylation of Akt, downstream P70S6, S6 and the expression of X-linked inhibitor of apoptosis protein (XIAP) [28], a physiologic substrate of Akt that is stabilized to inhibit programmed cell death (Figure 5B). We have previously shown that SASP-induced PEL apoptosis may also be orchestrated via reduction of intracellular GSH synthesis and increased ROS production [5]. Here we found that silencing of OSGINI significantly restored intracellular GSH synthesis and reduced ROS production from SASP-treated cells (Figure 5C-5D). Biochemical assays further confirmed that silencing of OSGIN1 caused a reduction in the activity of NADPH oxidase (Figure 5E), the major source of ROS production $[29,30]$. We also found that silencing of $\mathrm{xCT}$ by RNAi upregulated OSGIN1 transcripts in BCBL-1 cells, indicating that this gene is indeed a downstream target of $\mathrm{xCT}$ (Figure S3A). Taken together, these data demonstrate the central role of OSGIN1 in SASP-induced PEL apoptosis, which involves modulation of a variety of host physiologic factors.

We previously reported some other AIDS-related lymphoma cell-lines such as Burkitt's lymphoma BL-
A

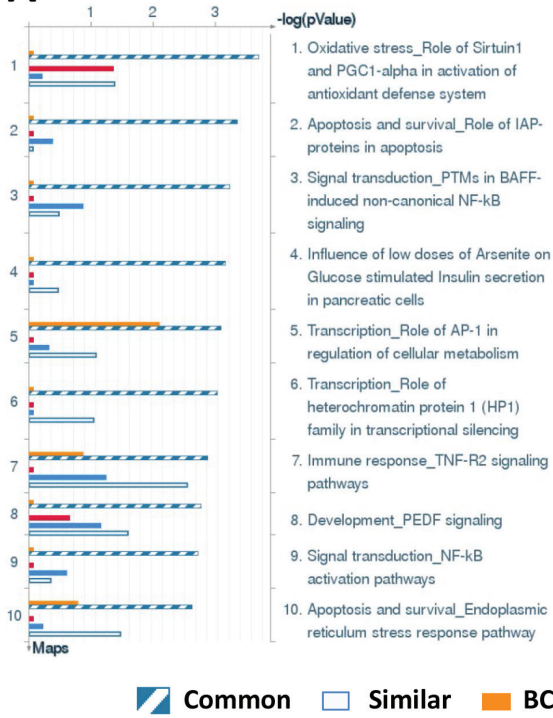

B

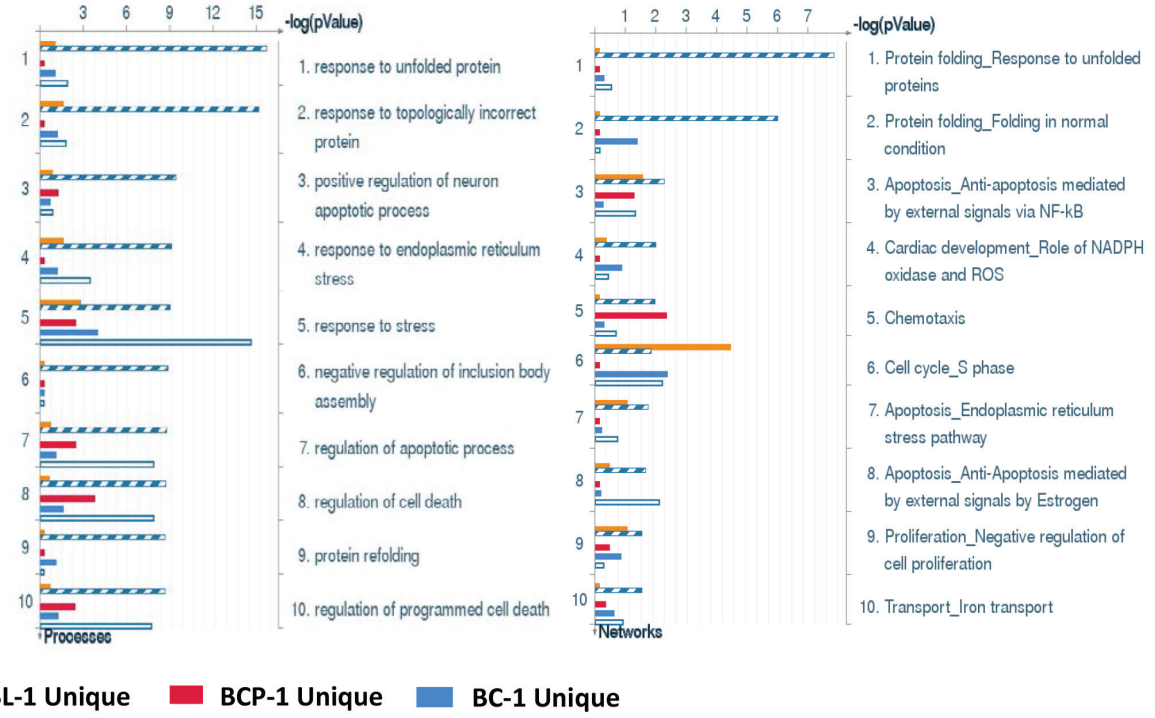

Figure 2: Enrichment analysis of gene profile significantly altered by inhibition of xCT within KSHV-infected PEL cell-lines. The enrichment analysis of gene profile (common, similar and unique profile as indicated) significantly altered by inhibition of xCT was performed using the Metacore Software (Thompson Reuters) Modules: Pathway Maps (A), Gene Ontology Processes (B) and Process Networks $(\mathbf{C})$. 

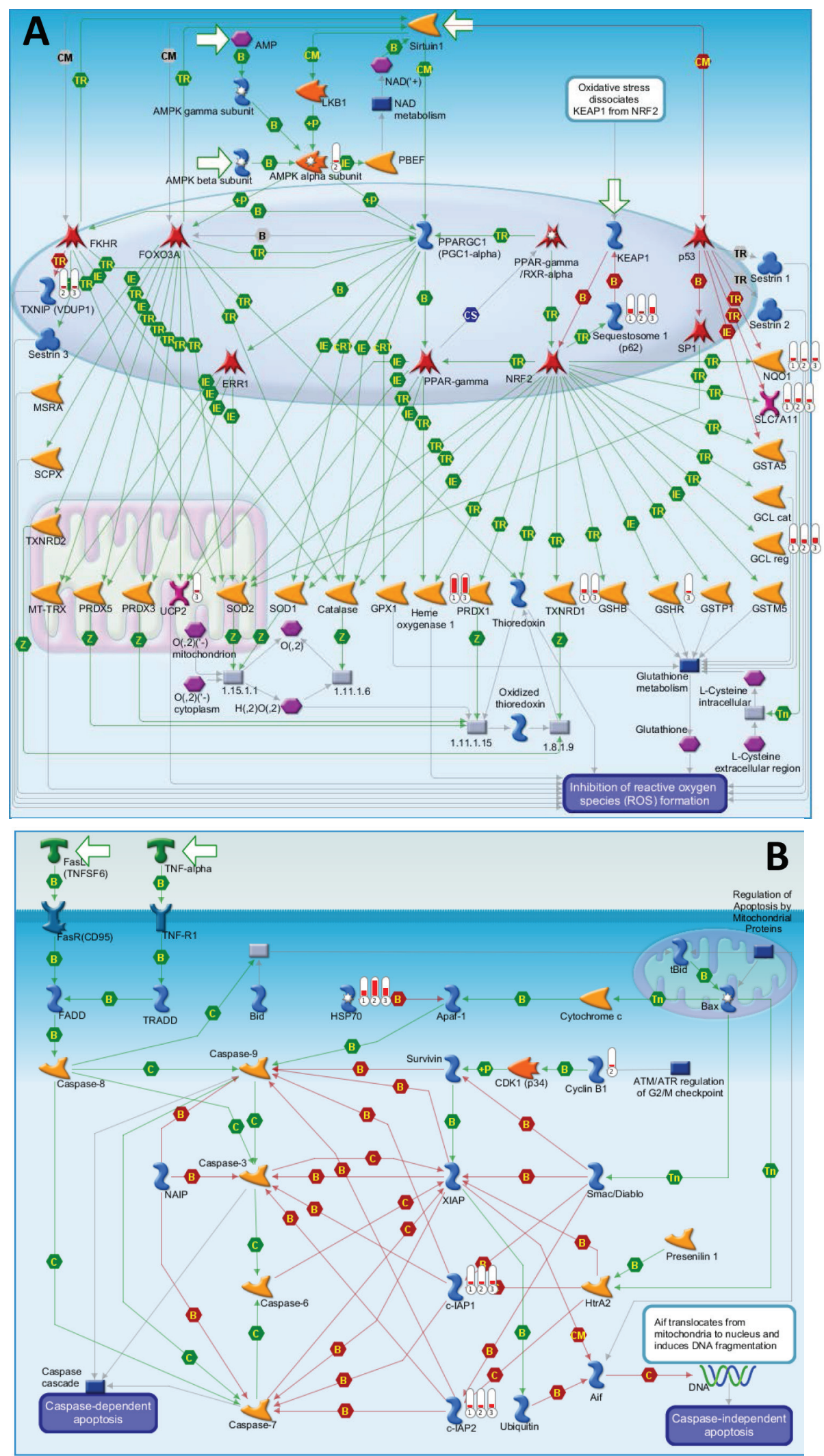

Figure 3: The top 2 scored maps (maps with the lowest $p$-value) based on the enrichment distribution sorted by 'common' gene set. (A) Oxidative stress: Role of Sirtuin1 and PGC1 alpha in activation of antioxidant defense system. (B) Apoptosis and survival: Role of IAP proteins in apoptosis. Experimental data from all files is linked to and visualized on the maps as thermometer like figures. Up-ward thermometers have red color and indicate upregulated signals and down ward (blue) ones indicate downregulated expression levels of the genes. Data was produced by the Metacore Software (Thompson Reuters). 
A

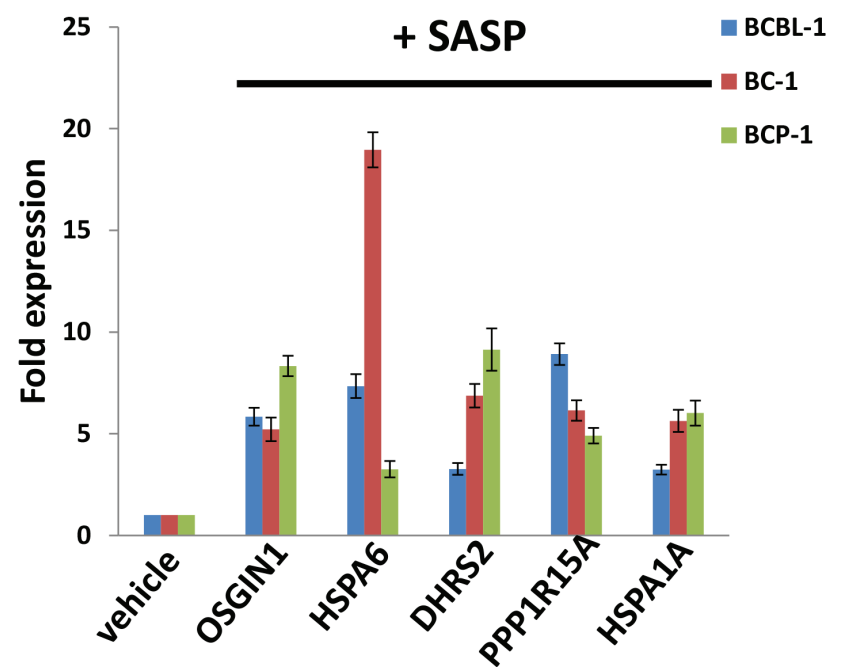

B

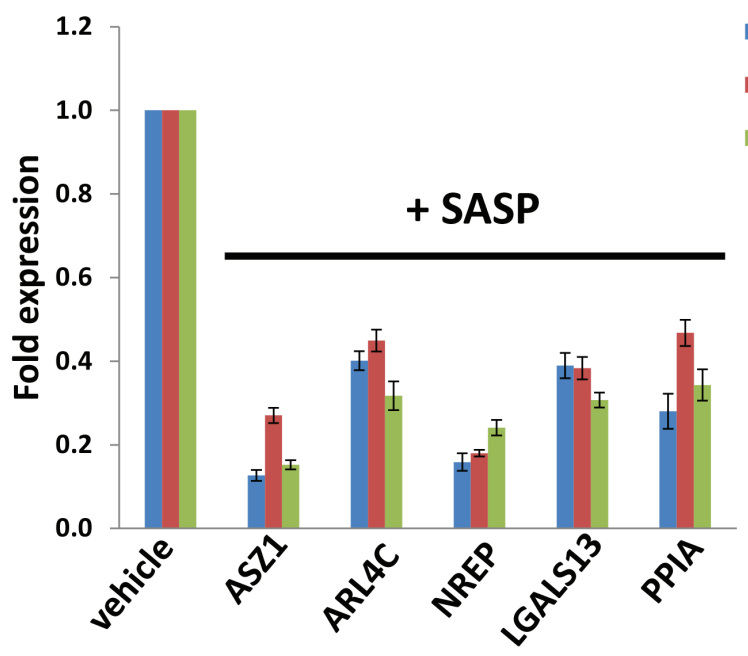

Figure 4: Validation of microarray results by qRT-PCR for selected candidate genes. The transcriptional levels of selected 5 candidate genes upregulated (A) or downregulated (B) as shown in microarray data were validated by using qRT-PCR, respectively. Error bars represent the S.E.M. for 3 independent experiments.
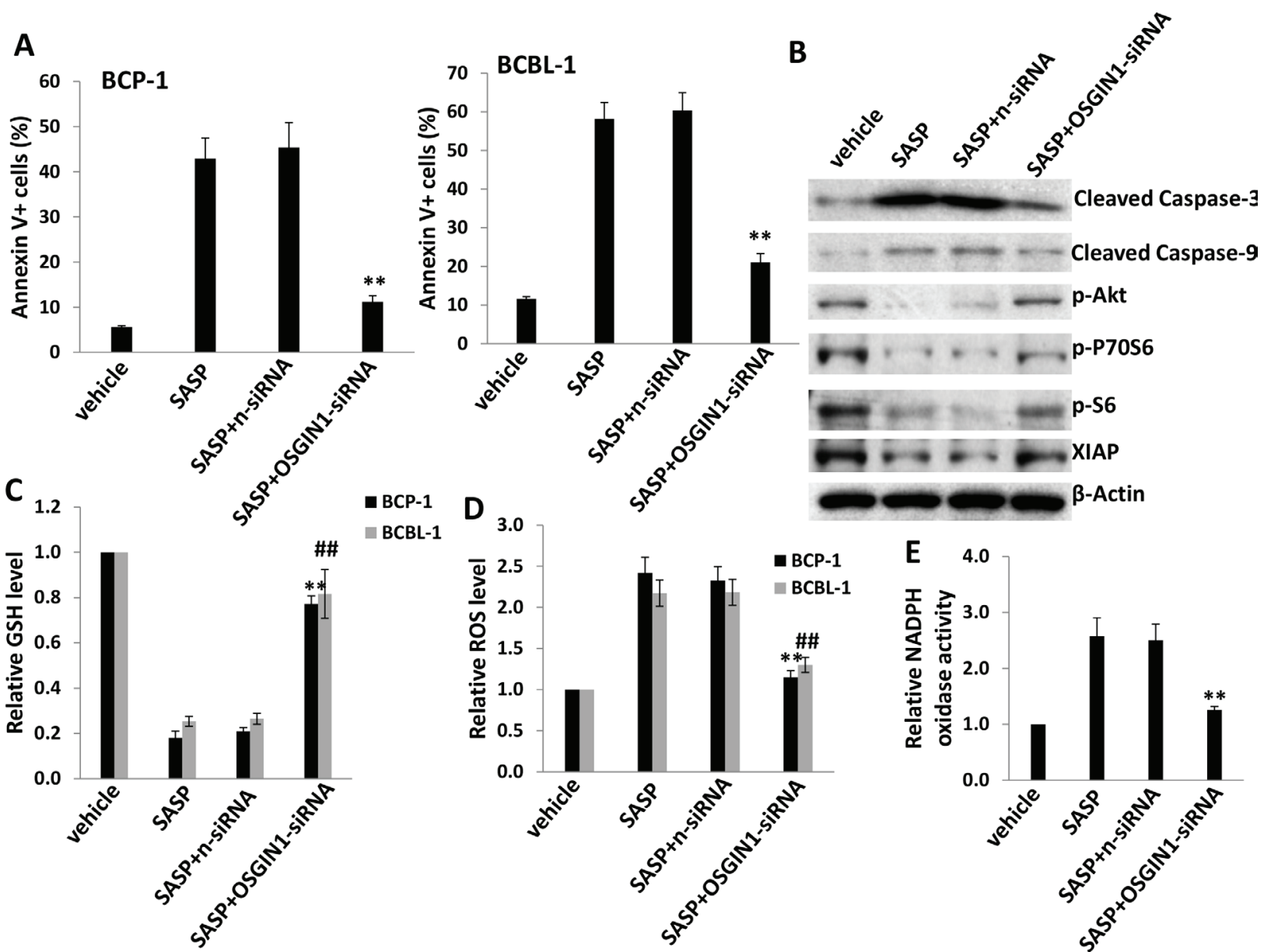

Figure 5: xCT inhibition induces KSHV-infected PEL cell apoptosis potentially through upregulation of OSGIN1. (A) BCP-1 and BCBL-1 were transfected with either negative control siRNA (n-siRNA) or OSGIN1-siRNA for $48 \mathrm{~h}$, then incubated with $0.5 \mathrm{mM}$ of SASP for additional $24 \mathrm{~h}$ and cell apoptosis was assessed using Annexin V-PI staining and flow cytometry analysis. (B) Protein expression in BCBL-1 was measured by immuoblots. (C-D) The levels of intracellular GSH and ROS were quantified as described in Methods. (E) The activities of NADPH oxidases in BCBL-1 were measured as described in Methods. Error bars represent the S.E.M. for 3 independent experiments. ${ }^{* *} / \# \#=p<0.01$ ( $v s \mathrm{SASP}+\mathrm{n}$-siRNA group). 
Table 2: The top 20 candidate genes downregulated in KSHV+ PEL cells treated by SASP.

\begin{tabular}{|c|c|c|c|c|}
\hline \multirow[t]{2}{*}{ Gene symbol } & \multirow[t]{2}{*}{ Description } & \multicolumn{3}{|c|}{ Fold change } \\
\hline & & BCP-1 & BC-1 & BCBL-1 \\
\hline ASZ1 & $\begin{array}{l}\text { Ankyrin repeat, SAM and basic leucine zipper domain-containing } \\
\text { protein } 1\end{array}$ & 0.31 & 0.19 & 0.32 \\
\hline ARL4C & ADP-ribosylation factor-like protein $4 \mathrm{C}$ & 0.25 & 0.33 & 0.25 \\
\hline NREP & Neuronal regeneration-related protein & 0.28 & 0.26 & 0.32 \\
\hline SOCS2 & Suppressor of cytokine signaling 2 & 0.28 & 0.23 & 0.39 \\
\hline FAM117B & Protein FAM117B & 0.33 & 0.23 & 0.4 \\
\hline LGALS13 & Galactoside-binding soluble lectin 13 & 0.32 & 0.22 & 0.44 \\
\hline PPIA & Peptidyl-prolyl cis-trans isomerase A & 0.3 & 0.26 & 0.43 \\
\hline BTF3L4 & Transcription factor BTF3 homolog 4 & 0.36 & 0.22 & 0.41 \\
\hline XRCC5 & X-ray repair cross-complementing protein 5 & 0.41 & 0.11 & 0.47 \\
\hline E2F5 & Transcription factor E2F5 & 0.35 & 0.31 & 0.35 \\
\hline ENPP2 & Ectonucleotide pyrophosphatase/phosphodiesterase family member & 0.32 & 0.28 & 0.41 \\
\hline PFKP & 6-phosphofructokinase type $\mathrm{C}$ & 0.35 & 0.29 & 0.38 \\
\hline RBM17 & Splicing factor 45 & 0.4 & 0.21 & 0.42 \\
\hline EPB41L3 & Band 4.1-like protein 3 & 0.36 & 0.3 & 0.38 \\
\hline ATP11B & Probable phospholipid-transporting ATPase IF & 0.3 & 0.35 & 0.4 \\
\hline SERBP1 & Plasminogen activator inhibitor 1 RNA-binding protein & 0.42 & 0.15 & 0.48 \\
\hline PM20D2 & Peptidase M20 domain-containing protein 2 & 0.47 & 0.21 & 0.37 \\
\hline MYLIP & E3 ubiquitin-protein ligase MYLIP & 0.21 & 0.39 & 0.47 \\
\hline CBR4 & Carbonyl reductase family member 4 & 0.38 & 0.22 & 0.47 \\
\hline TMPRSS3 & Transmembrane protease serine 3 & 0.3 & 0.32 & 0.46 \\
\hline
\end{tabular}

41 and $\mathrm{BJAB}$ (both are $\mathrm{KSHV}^{\text {neg }} / \mathrm{EBV}^{\text {neg }}$ ) with highly expressed $\mathrm{xCT}$, and SASP treatment induced significant apoptosis for BL-41 [5]. Here we found that silencing of OSGIN1 by siRNA significantly reduced cell apoptosis induced by SASP $(0.5 \mathrm{mM})$ in BL-41 cells (Figure S4). However, simply silencing of OSGIN1 did not induce apoptosis for primary human $\mathrm{CD}_{1} 9^{+} \mathrm{B}$ cells isolated from peripheral blood of healthy donor (Figure S5).
Targeting XRCC5 impairs DNA-damage repair abilities of tumor cells and promotes low dose of SASP-induced PEL apoptosis

We were also interested in XRCC5 (X-ray repair cross-complementing protein 5, also known as $\mathrm{Ku} 80$ ), one of downregulated genes in SASP-treated $\mathrm{KSHV}^{+} \mathrm{PEL}$ cells, to determine its role in SASP-induced cell apoptosis. $\mathrm{Ku} 80$ is a tightly associated heterodimer of $\sim 70 \mathrm{kDa}$ and $\sim 80 \mathrm{kDa}$ subunits (Ku70 and Ku80) that, together with the $\sim 470 \mathrm{kDa}$ catalytic subunit, DNA-PKcs, form the DNA-dependent protein kinase involved in repairing DNA 
double-strand breaks (DSBs) caused by a variety of stress factors [31]. The Ku80-dependent repair process, called nonhomologous end joining (NHEJ), appears to be the main DNA DSB repair mechanism in mammalian cells [31, 32]. Interestingly, Ku80-knockout mice are small, and their cells fail to proliferate in culture and show signs of premature senescence $[33,34]$. Here we found, for the first time, that silence of $\mathrm{xCT}$ by RNAi significantly downregulates XRCC5 (Ku80) transcripts in BCBL-1 cells, indicating that $X R C C 5$ is also a downstream gene target of $\mathrm{xCT}$ (Figure S3B). Interestingly, direct siRNA silencing of XRCC5 enhanced low-dose SASP (0.1mM)induced PEL apoptosis, potentially due to impaired DNA-damage repair machinery in tumor cells (Figures 6A and S6). Immunoblot and immunofluorescence data further confirmed that silencing of XRCC5 in low dose SASP-treated BCBL-1 cells increased the levels of cleaved Caspase 3 and 9 as well as phosphorylated p53
(Ser15) and phosphorylated Histone H2A.X (Ser139), the two markers for DNA-damage [35] (Figure 6B and 6C). Moreover, we found that silencing of XRCC5 also enhanced the induction of apoptosis and programmed cell death by low dose concentrations of other DNA-damage reagents such as Doxorubicin $(100 \mathrm{nM})$ induced PEL apoptosis (Figure 6D and 6E). Together, these data provide solid evidence that DNA-damage may represent another mechanism of SASP-induced PEL apoptosis/cell death, which is potentially through XRCC5. In addition, direct siRNA silencing of XRCC5 also enhanced low-dose SASP (0.1 mM)-induced apoptosis for Burkitt's lymphoma BL41 cells (Figure $\mathrm{S} 7$ ), while targeting XRCC5 induced no apoptosis for primary human $\mathrm{CD} 19^{+} \mathrm{B}$ cells isolated from peripheral blood of healthy donor (Figure S5).
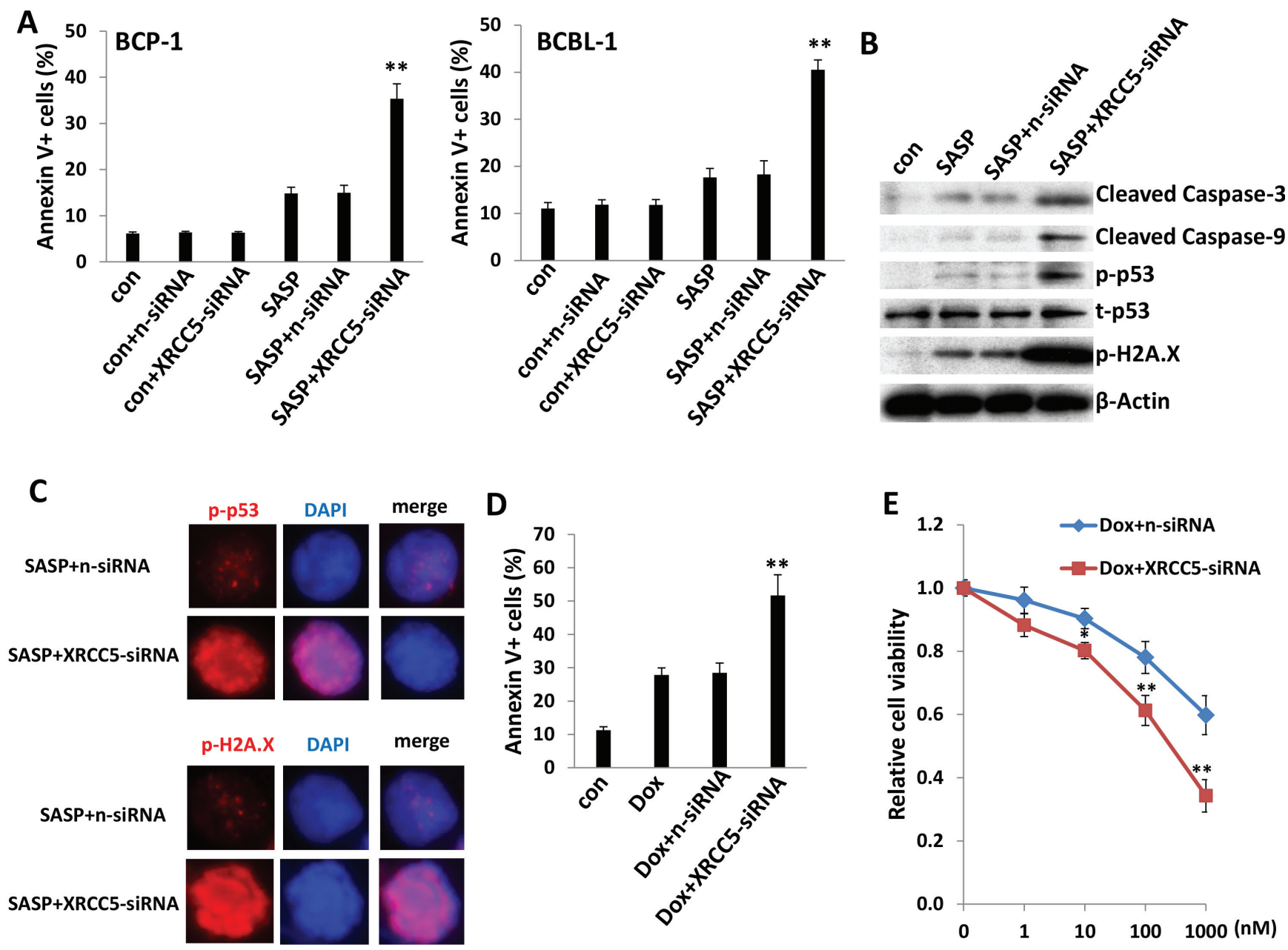

Figure 6: Targeting XRCC5 impairs DNA-damage repair abilities of PEL cells and promotes chemicals-induced apoptosis. (A) BCP-1 and BCBL-1 were transfected with either negative control siRNA (n-siRNA) or XRCC5-siRNA for 48 h, then some cells incubated with $0.1 \mathrm{mM}$ of SASP for additional $24 \mathrm{~h}$ and cell apoptosis was assessed using Annexin V-PI staining and flow cytometry analysis. (B-C) Protein expression in BCBL-1 was measured by immuoblots and immunofluorescence, respectively. (D-E) BCBL-1 were transfected as (A), then incubated with $100 \mathrm{nM}$ of doxorubicin (Dox) or indicated concentrations for additional $48 \mathrm{~h}$, then cell apoptosis and death were measured by flow cytometry and MTT assay, respectively. Error bars represent the S.E.M. for 3 independent experiments. $*=p<0.05, * *=p<0.01$ ( vs SASP+n-siRNA group or Dox $+\mathrm{n}-\mathrm{siRNA})$. 


\section{DISCUSSION}

Recent studies have demonstrated that KSHV contributes to PEL survival and proliferation in part by downregulating or inactivating specific tumor suppressor genes. For example, KSHV-encoded latency-associated nuclear antigen (LANA) can directly interact with and inactivate the tumor suppressor functions of $\mathrm{p} 53$ and $\mathrm{p} 73$, thus promoting tumor cell survival $[36,37]$. In addition, one of the KSHV microRNAs, miR-K12-1, directly targets and represses expression of $\mathrm{p} 21$, a well-known cyclindependent kinase (CDK) inhibitor, to promote PEL cell growth [38]. Furthermore, the amino acid transporter, $\mathrm{xCT}$, which is highly expressed on the surface of $\mathrm{KSHV}^{+}$ PEL cells, also supports PEL growth not only through its amino-acid transport function but also through regulation of downstream cell survival effectors [5]. This is supported by our previous data demonstrating that the $\mathrm{xCT}$ selective inhibitor, SASP, blocks PEL tumor progression in an immune-deficient xenograft model [5], suggesting that the infectious process of KSHV is directly linked to post-entry mechanisms involved in virus-associated lymphomagenesis, but the mechanisms by which $\mathrm{xCT}$ orchestrates this link are not fully defined.

In the current study, we used microarray analysis to interrogate the transcriptional profile of SASP-treated $\mathrm{KSHV}^{+} \mathrm{PEL}$ cell lines, and identified a number of genes whose expression was altered in a unique and global manner. Enrichment analysis indicated that targeting xCT in this manner resulted in upregulated expression of a class of genes that may function to promote PEL cell survival in part by preventing apoptosis and/or programmed cell death, implying that in addition to its natural function as amino acid transporter, $\mathrm{xCT}$ also acts as a global regulator of down-stream effector proteins involved in tumor cell survival.

First we discovered that OSGIN1, a tumor suppressor gene that is upregulated in SASP-treated PEL cells, plays a role in SASP-induced PEL apoptosis through regulation of Akt signaling, GSH synthesis and ROS production. Interestingly, the OSGIN1 homolog, bone marrow stromal cell (BMSC)-derived growth inhibitor (BDGI), has been shown to induce cell cycle arrest in $\mathrm{S}$ phase and subsequent apoptosis of MCF-7 breast cancer cells, which potentially occurs through upregulation of $\mathrm{p} 27^{\mathrm{Kipl}}$ and downregulation of cyclin $\mathrm{A}, \mathrm{Bcl}-2$, and Bcl-xL [39]. Therefore, our data are consistent with an emerging theme with respect to xCT-mediated tumor cell survival, and sets the stage for derivative studies aimed at determining whether targeting the $\mathrm{xCT} / \mathrm{OSGIN} 1$ axis will also impact proteins involved in the PEL cell cycle.

Doxorubicin, a DNA-damage reagent, is one of the first-line chemotherapy drugs for PEL treatment [3]. However, our previous studies have demonstrated that some $\mathrm{KSHV}^{+}$PEL cell-lines (e.g. BCP-1 and BCBL1) display multidrug chemoresistance to a number of chemotherapeutic drugs, including Doxorubicin [40]. Here we found that siRNA "knock-down" of XRCC5, one of the downstream genes regulated by $\mathrm{xCT}$, impaired the DNA-damage repair machinery and sensitized BCBL-1 to low dose Doxorubicin-induced cell apoptosis/cell death. This result is consistent with a previous study in which Huang et al. demonstrated a link between the level of $\mathrm{xCT}$ expression in a panel of cancer cell lines with the potency of 1,400 candidate anticancer drugs, with 39 positive correlations, and 296 negative correlations [41]. Therefore, we have reason to believe that targeting $\mathrm{xCT} /$ XRCC5 represents a promising "combination" strategy for enhancing the efficacy of chemotherapeutic drugs while reducing systemic cytotoxicity.

Considered in a broader context, our data supports evaluation of $\mathrm{xCT}$ targeting as a means to attenuate survival and/or growth of other non-KSHV-associated lymphomas as well. For instance, $\mathrm{xCT}$ is expressed on some Burkitt's lymphoma cell-lines such as BL-41 and BJAB (both KSHV $\left.{ }^{\text {neg }} / \mathrm{EBV}^{\text {neg }}\right)$, AKATA $\left(\mathrm{KSHV}^{\text {neg }} / \mathrm{EBV}^{+}\right)$ and on some diffuse large cell lymphoma (DLCL) celllines such as CRL2631 ( $\left.\mathrm{KSHV}^{\text {neg }} / \mathrm{EBV}^{\text {neg }}\right)$, and inhibition of $\mathrm{xCT}$ by SASP also induced significant apoptosis in BL-41 lymphoma cells expressing high levels of xCT [5]. Therefore, it will be interesting to explore the global gene profile altered by targeting $\mathrm{xCT}$ in other AIDS-related lymphoma cells as well. As mentioned above, SASP treatment induced a much higher number of uniquely altered genes in the dually infected $\mathrm{BC}-1\left(\mathrm{KSHV}^{+} / \mathrm{EBV}^{+}\right)$ cells than in BCBL-1 and BCP-1 (both of which are $\mathrm{KSHV}^{+} / \mathrm{EBV}^{\text {neg }}$ ), suggesting that complex interactions between these co-existent oncogenic herpesviruses may influence the outcome of SASP treatment. In this respect subtractive microarray analysis of differential gene expression cell lines latently infected with one or both viruses may reveal important themes related to their strategies for persistence and induction of associated malignancies.

\section{MATERIALS AND METHODS}

\section{Cell culture and reagents}

The PEL cell-line BCBL-1 $\left(\mathrm{KSHV}^{+} / \mathrm{EBV}^{\text {neg }}\right)$ and a Burkitt's lymphoma cell line BL-41 (KSHV $\left.{ }^{\text {neg }} / \mathrm{EBV}^{\text {neg }}\right)$ was kindly provided by Dr. Dean Kedes (University of Virginia) and maintained in RPMI 1640 medium (Gibco) with supplements as described previously [42]. The other PEL cell-lines BC-1 $\left(\mathrm{KSHV}^{+} / \mathrm{EBV}^{+}\right)$and $\mathrm{BCP}-1\left(\mathrm{KSHV}^{+} /\right.$ $\mathrm{EBV}^{\mathrm{neg}}$ ) were purchased from American Type Culture Collection (ATCC) and maintained in complete RPMI 1640 medium (ATCC) supplemented with 20\% FBS. All cells were cultured at $37^{\circ} \mathrm{C}$ in $5 \% \mathrm{CO}_{2}$. All experiments were carried out using cells harvested at low $(<20)$ 
passages. Sulfasalazine (SASP) and Doxorubicin were purchased from Sigma.

\section{Microarray}

Microarray analysis was performed and analyzed at the Stanley S. Scott Cancer Center's Translational Genomics Core at LSUHSC. BC-1, BCP-1 and BCBL1 cells were treated with vehicle or the $\mathrm{xCT}$ selective inhibitor SASP $(0.5 \mathrm{mM})$ for $48 \mathrm{~h}$, respectively. Total RNA was isolated using Qiagen RNeasy kit (Qiagen), and $500 \mathrm{ng}$ of total RNA was used to synthesize dscDNA. Biotin-labeled RNA was generated using the TargetAmpNano Labeling Kit for Illumina Expression BeadChip (Epicentre), according to the manufacturers' instructions, and hybridized to the HumanHT-12 v4 Expression BeadChip (Illumina) which contains more than 47,000 probes derived from the NCBI RefSeq Release 38 and other sources, at $58^{\circ} \mathrm{C}$ for $16 \mathrm{~h}$. The chip was washed, stained with streptavadin-Cy3, and scanned with the Illumina BeadStation 500 and BeadScan. Using the Illumina's GenomeStudio software, we normalized the signals using the "cubic spline algorithm" that assumes that the distribution of the transcript abundance is similar in all samples, according to the method proposed by Workman et al. [43]. The background signal was removed using the "detection $p$-value algorithm" to remove targets with signal intensities equal or lower than that of irrelevant probes (with no known targets in the human genome but thermodynamically similar to the relevant probes). The microarray experiments were performed twice for each group and the average values were used for analysis. Common, similar, and unique sets of genes and enrichment analysis were performed using the MetaCore Software (Thompson Reuters) as previously reported [23]. The microarray original data have been submitted to Gene Expression Omnibus (GEO) database (Accession number: GSE65418).

\section{Isolation of circulating human B cells}

Human peripheral blood mononuclear cells (PBMC) were isolated from whole blood from the healthy donor following Ficoll gradient separation. PBMC were washed and resuspended in $500 \mu \mathrm{L}$ total volume, including 440 $\mu \mathrm{L}$ buffer composed of $2 \%$ FBS and $1 \mathrm{mM}$ EDTA in 1X PBS (EasySep buffer, STEMCELL Technologies), $30 \mu \mathrm{L}$ Fc-receptor blocker (eBiosciences), and $30 \mu \mathrm{L}$ of a PE-conjugated anti-CD19 monoclonal antibody (BD-Pharmagen), for incubation at RT for 20 minutes. $100 \mu \mathrm{L}$ EasySep PE selection cocktail (STEMCELL Technologies) was added for an additional 15 minutes, and $2.5 \mathrm{~mL}$ of additional buffer was then added prior to magnetic column separation of $\mathrm{CD} 19^{+}$cells. Following column separation, supernatants were discarded and cells resuspended in fresh $2.5 \mathrm{~mL}$ buffer for each of two additional column separation steps. Thereafter, cells were resuspended in complete RPMI 1640 medium supplemented with $20 \%$ FBS for further experiments, or in 1X PBS for flow cytometry to determine the purity of selection. $92-95 \%$ pure populations of $\mathrm{CD} 19^{+}$cells were recovered (data not shown).

\section{Cell viability assays}

Cell viability was assessed using MTT assays for assessment of proliferative capacity, and flow cytometry was used for quantitative assessment of apoptosis. Standard MTT assays were performed as described previously [5]. For flow cytometry, the FITC-Annexin V/propidium iodide (PI) Apoptosis Detection Kit I (BD Pharmingen) was used according to the manufacturer's instructions.

\section{Immunoblotting}

Cells were lysed in buffer containing $20 \mathrm{mM}$ Tris (pH 7.5), $150 \mathrm{mM} \mathrm{NaCl}, 1 \%$ NP40, 1 mM EDTA, $5 \mathrm{mM}$ $\mathrm{NaF}$ and $5 \mathrm{mM} \mathrm{Na}_{3} \mathrm{VO}_{4}$. Total cell lysates $(30 \mu \mathrm{g})$ were resolved by $10 \%$ SDS-PAGE, transferred to nitrocellulose membranes, and immunoblotted using 100-200 $\mu \mathrm{g} / \mathrm{mL}$ antibodies to cleaved-caspase 3/9, p-Akt, p-P70S6, p-S6, p-H2A.X, p-p53/t-p53, and XIAP (all purchased from Cell Signaling, Inc., Danvers, MA). For loading controls, blots were incubated with antibodies detecting $\beta$-Actin (Sigma). Immunoreactive bands were developed using an enhanced chemiluminescence reaction (Perkin-Elmer) and visualized by autoradiography.

\section{Immunofluorescence assays (IFA)}

Cells were incubated in 1:1 methanol-acetone at $-20^{\circ} \mathrm{C}$ for fixation and permeabilization, then with a blocking reagent $(10 \%$ normal goat serum, $3 \%$ bovine serum albumin, and $1 \%$ glycine) for an additional 30 minutes. Cells were then incubated for $1 \mathrm{~h}$ at $25^{\circ} \mathrm{C}$ with 1:400 dilution of a mouse anti-p-p53 antibody or a rabbit anti-p-H2A.X antibody (Cell Signaling) followed by 1:200 dilution of a goat anti-mouse or goat antirabbit secondary antibody conjugated with Texas Red (Invitrogen), respectively. For identification of nuclei, cells were subsequently counterstained with $0.5 \mu \mathrm{g} / \mathrm{mL}$ 4',6-diamidino-2-phenylindole (DAPI; Sigma) in $180 \mathrm{mM}$ Tris- $\mathrm{HCl}$ (pH 7.5). Cells were washed once in $180 \mathrm{mM}$ Tris- $\mathrm{HCl}$ for 15 minutes and prepared for visualization using a Leica TCPS SP5 AOBS confocal microscope. 


\section{RNA interference}

For RNA interference assays, ON-TARGET plus SMART pool siRNA for $x C T$, OSGIN1 or XRCC5 (Dharmacon), or negative control siRNA, were delivered using the DharmaFECT transfection reagent according to the manufacturer's instructions.

\section{qRT-PCR}

Total RNA was isolated using the RNeasy Mini kit according to the manufacturer's instructions (QIAGEN). cDNA was synthesized from equivalent total RNA using SuperScript III First-Strand Synthesis SuperMix Kit (Invitrogen) according to the manufacturer's procedures. Primers used for amplification of target genes are displayed in Table S1. Amplification was carried out using an iCycler IQ Real-Time PCR Detection System, and cycle threshold $(\mathrm{Ct})$ values were tabulated in duplicate for each gene of interest in each experiment. "No template" (water) controls were used to ensure minimal background contamination. Using mean $\mathrm{Ct}$ values tabulated for each gene, and paired $\mathrm{Ct}$ values for $\beta$-actin as an internal control, fold changes for experimental groups relative to assigned controls were calculated using automated iQ5 2.0 software (Bio-rad).

\section{ROS measurement}

PEL cells were loaded with $10 \mu \mathrm{M}$ of the ROS dye c-H2DCFDA (Invitrogen) for $30 \mathrm{~min}$ at $37^{\circ} \mathrm{C}$ in Hanks' Balanced Salt Solution (HBSS) containing calcium and magnesium (HBSS $/ \mathrm{Ca} / \mathrm{Mg}$ ). Cells were then washed once with $\mathrm{HBSS} / \mathrm{Ca} / \mathrm{Mg}$ to remove dye, resuspended in $\mathrm{HBSS} / \mathrm{Ca} / \mathrm{Mg}$ and subjected to flow cytometry analysis as previously described [5].

\section{NADPH oxidase activities assays}

The chemiluminescence-based NADPH oxidase activity assays were performed as described previously [5]. After drug-treatment, cells were centrifuged at $500 \mathrm{~g}$ for $10 \mathrm{~min}$ at $4^{\circ} \mathrm{C}$. The cell pellet was resuspended in 35 $\mu \mathrm{L}$ ice-cold lysis buffer and kept on ice for $20 \mathrm{~min}$. To a final $200 \mu \mathrm{L}$ of $\mathrm{HBSS} / \mathrm{Ca} / \mathrm{Mg}$ buffer containing NADPH ( $1 \mu \mathrm{M}$, Sigma) and lucigenin ( $20 \mu \mathrm{M}$, Sigma $), 5 \mu \mathrm{L}$ of cell lysates was added to initiate the reaction for $5 \mathrm{~min}$ at $37^{\circ} \mathrm{C}$. Chemiluminescence was measured immediately using a Synergy HT microplate reader (BioTek Instruments).

\section{Intracellular GSH measurement}

The intracellular GSH levels in PEL cells were quantified using the GSH-Glo ${ }^{\mathrm{TM}}$ Glutathione Assay Kit (Promega), according to the manufacturer's instructions.

\section{Statistical analyses}

Significance for differences between experimental and control groups was determined using the two-tailed Student's t-test (Excel 8.0).

\section{ACKNOWLEDGMENTS}

This work was supported by grants from a Center for Biomedical Research Excellence P20-GM103501 subaward (RR021970), the DOD Career Development Award (CA140437), the Ladies Leukemia League Grant (2014-2015) and the National Natural Science Foundation (NNSF) of China (81101791, 81272191, 81472547 and 81400164). The funders had no role in study design, data collection and analysis, decision to publish, or preparation of the manuscript.

\section{COMPETING INTERESTS}

The authors declare that they have no competing interests.

\section{REFERENCES}

1. Cesarman E, Chang Y, Moore PS, Said JW and Knowles DM. Kaposi's sarcoma-associated herpesvirus-like DNA sequences in AIDS-related body-cavity-based lymphomas. N Engl J Med. 1995; 332:1186-1191.

2. Simonelli C, Spina M, Cinelli R, Talamini R, Tedeschi R, Gloghini A, Vaccher E, Carbone A and Tirelli U. Clinical features and outcome of primary effusion lymphoma in HIV-infected patients: a single-institution study. J Clin Oncol. 2003; 21:3948-3954.

3. Chen YB, Rahemtullah A and Hochberg E. Primary effusion lymphoma. Oncologist. 2007; 12:569-576.

4. Boulanger E, Gerard L, Gabarre J, Molina JM, Rapp C, Abino JF, Cadranel J, Chevret S and Oksenhendler E. Prognostic factors and outcome of human herpesvirus 8 -associated primary effusion lymphoma in patients with AIDS. J Clin Oncol. 2005; 23:4372-4380.

5. Dai L, Cao Y, Chen Y, Parsons C and Qin Z. Targeting $\mathrm{xCT}$, a cystine-glutamate transporter induces apoptosis and tumor regression for KSHV/HIV-associated lymphoma. J Hematol Oncol. 2014; 7:30.

6. Bannai S. Exchange of cystine and glutamate across plasma membrane of human fibroblasts. J Biol Chem. 1986; 261:2256-2263. 
7. Patel SA, Warren BA, Rhoderick JF and Bridges RJ. Differentiation of substrate and non-substrate inhibitors of transport system $\mathrm{xc}(-)$ : an obligate exchanger of L-glutamate and L-cystine. Neuropharmacology. 2004; 46:273-284

8. Gout PW, Buckley AR, Simms CR and Bruchovsky N. Sulfasalazine, a potent suppressor of lymphoma growth by inhibition of the $\mathrm{x}(\mathrm{c})$ - cystine transporter: a new action for an old drug. Leukemia. 2001; 15:1633-1640.

9. Chung WJ, Lyons SA, Nelson GM, Hamza H, Gladson CL, Gillespie GY and Sontheimer H. Inhibition of cystine uptake disrupts the growth of primary brain tumors. J Neurosci. 2005; 25:7101-7110.

10. Narang VS, Pauletti GM, Gout PW, Buckley DJ and Buckley AR. Suppression of cystine uptake by sulfasalazine inhibits proliferation of human mammary carcinoma cells. Anticancer Res. 2003; 23:4571-4579.

11. Doxsee DW, Gout PW, Kurita T, Lo M, Buckley AR, Wang Y, Xue H, Karp CM, Cutz JC, Cunha GR and Wang YZ. Sulfasalazine-induced cystine starvation: potential use for prostate cancer therapy. Prostate. 2007; 67:162-171.

12. Kaleeba JA and Berger EA. Kaposi's sarcoma-associated herpesvirus fusion-entry receptor: cystine transporter $\mathrm{xCT}$. Science. 2006; 311:1921-1924.

13. Veettil MV, Sadagopan S, Sharma-Walia N, Wang FZ, Raghu H, Varga L and Chandran B. Kaposi's sarcomaassociated herpesvirus forms a multimolecular complex of integrins (alphaVbeta5, alphaVbeta3, and alpha3beta1) and CD98-xCT during infection of human dermal microvascular endothelial cells, and CD98-xCT is essential for the postentry stage of infection. J Virol. 2008; 82:12126-12144.

14. Chang Y, Cesarman E, Pessin MS, Lee F, Culpepper J, Knowles DM and Moore PS. Identification of herpesviruslike DNA sequences in AIDS-associated Kaposi's sarcoma. Science. 1994; 266:1865-1869.

15. Qin Z, Freitas E, Sullivan R, Mohan S, Bacelieri R, Branch D, Romano M, Kearney P, Oates J, Plaisance K, Renne R, Kaleeba J and Parsons C. Upregulation of xCT by KSHVencoded microRNAs facilitates KSHV dissemination and persistence in an environment of oxidative stress. PLoS Pathog. 2010; 6:e1000742.

16. Qin Z, Dai L, Defee M, Findlay VJ, Watson DK, Toole BP, Cameron J, Peruzzi F, Kirkwood K and Parsons C. Kaposi's Sarcoma-Associated Herpesvirus Suppression of DUSP1 Facilitates Cellular Pathogenesis following De Novo Infection. J Virol. 2013; 87:621-635.

17. Bridges $\mathrm{CC}, \mathrm{Hu} \mathrm{H}$, Miyauchi S, Siddaramappa UN, Ganapathy ME, Ignatowicz L, Maddox DM, Smith SB and Ganapathy V. Induction of cystine-glutamate transporter xc- by human immunodeficiency virus type 1 transactivator protein tat in retinal pigment epithelium. Invest Ophthalmol Vis Sci. 2004; 45:2906-2914.

18. Sato H, Fujiwara K, Sagara J and Bannai S. Induction of cystine transport activity in mouse peritoneal macrophages by bacterial lipopolysaccharide. Biochem J. 1995; 310 :547551.

19. Sato H, Kuriyama-Matsumura K, Hashimoto T, Sasaki $\mathrm{H}$, Wang H, Ishii T, Mann GE and Bannai S. Effect of oxygen on induction of the cystine transporter by bacterial lipopolysaccharide in mouse peritoneal macrophages. J Biol Chem. 2001; 276:10407-10412.

20. Taguchi K, Tamba M, Bannai S and Sato H. Induction of cystine/glutamate transporter in bacterial lipopolysaccharide induced endotoxemia in mice. J Inflamm (Lond). 2007; 4:20.

21. Pozzesi N, Pierangeli S, Vacca C, Falchi L, Pettorossi V, Martelli MP, Thuy TT, Ninh PT, Liberati AM, Riccardi C, Sung TV and Delfino DV. Maesopsin 4-O-beta-Dglucoside, a natural compound isolated from the leaves of Artocarpus tonkinensis, inhibits proliferation and upregulates HMOX1, SRXN1 and BCAS3 in acute myeloid leukemia. J Chemother. 2011; 23:150-157.

22. Moon JS, Kim HE, Koh E, Park SH, Jin WJ, Park BW, Park SW and Kim KS. Kruppel-like factor 4 (KLF4) activates the transcription of the gene for the platelet isoform of phosphofructokinase (PFKP) in breast cancer. J Biol Chem. 2011; 286:23808-23816.

23. Kim SH, Sierra RA, McGee DJ and Zabaleta J. Transcriptional profiling of gastric epithelial cells infected with wild type or arginase-deficient Helicobacter pylori. BMC Microbiol. 2012; 12:175.

24. Huynh $\mathrm{H}, \mathrm{Ng} \mathrm{CY}$, Ong CK, Lim KB and Chan TW. Cloning and characterization of a novel pregnancy-induced growth inhibitor in mammary gland. Endocrinology. 2001; 142:3607-3615.

25. Ong CK, Leong C, Tan PH, Van T and Huynh $\mathrm{H}$. The role of 5' untranslated region in translational suppression of OKL38 mRNA in hepatocellular carcinoma. Oncogene. 2007; 26:1155-1165.

26. Liu M, Li Y, Chen L, Chan TH, Song Y, Fu L, Zeng TT, Dai YD, Zhu YH, Li Y, Chen J, Yuan YF and Guan XY. Allelespecific imbalance of oxidative stress-induced growth inhibitor 1 associates with progression of hepatocellular carcinoma. Gastroenterology. 2014; 146:1084-1096.

27. Ong CK, Ng CY, Leong C, Ng CP, Foo KT, Tan PH and Huynh H. Genomic structure of human OKL38 gene and its differential expression in kidney carcinogenesis. J Biol Chem. 2004; 279:743-754.

28. Dan HC, Sun M, Kaneko S, Feldman RI, Nicosia SV, Wang HG, Tsang BK and Cheng JQ. Akt phosphorylation and stabilization of X-linked inhibitor of apoptosis protein (XIAP). J Biol Chem. 2004; 279:5405-5412.

29. Lambeth JD. NOX enzymes and the biology of reactive oxygen. Nat Rev Immunol. 2004; 4:181-189.

30. Bedard $\mathrm{K}$ and Krause KH. The NOX family of ROS-generating NADPH oxidases: physiology and pathophysiology. Physiol Rev. 2007; 87:245-313.

31. Featherstone $\mathrm{C}$ and Jackson $\mathrm{SP}$. Ku, a DNA repair protein 
with multiple cellular functions? Mutat Res. 1999; 434:315 .

32. Grundy GJ, Moulding HA, Caldecott KW and Rulten SL. One ring to bring them all--the role of $\mathrm{Ku}$ in mammalian non-homologous end joining. DNA Repair (Amst). 2014; 17:30-38.

33. Nussenzweig A, Chen C, da Costa Soares V, Sanchez M, Sokol K, Nussenzweig MC and Li GC. Requirement for $\mathrm{Ku} 80$ in growth and immunoglobulin V(D)J recombination. Nature. 1996; 382:551-555.

34. Zhu C, Bogue MA, Lim DS, Hasty P and Roth DB. Ku86deficient mice exhibit severe combined immunodeficiency and defective processing of $\mathrm{V}(\mathrm{D}) \mathrm{J}$ recombination intermediates. Cell. 1996; 86:379-389.

35. Peraldo-Neia C, Cavalloni G, Soster M, Gammaitoni L, Marchio S, Sassi F, Trusolino L, Bertotti A, Medico E, Capussotti L, Aglietta M and Leone F. Anti-cancer effect and gene modulation of ET-743 in human biliary tract carcinoma preclinical models. BMC Cancer. 2014; 14:918.

36. Chen W, Hilton IB, Staudt MR, Burd CE and Dittmer DP. Distinct p53, p53:LANA, and LANA complexes in Kaposi's Sarcoma--associated Herpesvirus Lymphomas. J Virol. 2010; 84:3898-3908.

37. Santag S, Jager W, Karsten CB, Kati S, Pietrek M, Steinemann D, Sarek G, Ojala PM and Schulz TF. Recruitment of the tumour suppressor protein $\mathrm{p} 73$ by Kaposi's Sarcoma Herpesvirus latent nuclear antigen contributes to the survival of primary effusion lymphoma cells. Oncogene. 2013; 32:3676-3685.

38. Gottwein E and Cullen BR. A human herpesvirus microRNA inhibits p21 expression and attenuates p21mediated cell cycle arrest. J Virol. 2010; 84:5229-5237.

39. Wang T, Xia D, Li N, Wang C, Chen T, Wan T, Chen $\mathrm{G}$ and Cao X. Bone marrow stromal cell-derived growth inhibitor inhibits growth and migration of breast cancer cells via induction of cell cycle arrest and apoptosis. J Biol Chem. 2005; 280:4374-4382.

40. Qin Z, Dai L, Bratoeva M, Slomiany MG, Toole BP and Parsons C. Cooperative roles for emmprin and LYVE-1 in the regulation of chemoresistance for primary effusion lymphoma. Leukemia. 2011; 25:1598-1609.

41. Huang Y, Dai Z, Barbacioru C and Sadee W. Cystineglutamate transporter SLC7A11 in cancer chemosensitivity and chemoresistance. Cancer Res. 2005; 65:7446-7454.

42. Dai L, Trillo-Tinoco J, Bai L, Kang B, Xu Z, Wen X, Del Valle L and Qin Z. Systematic analysis of a xenograft mice model for $\mathrm{KSHV}+$ primary effusion lymphoma (PEL). PLoS One. 2014; 9:e90349.

43. Workman C, Jensen LJ, Jarmer H, Berka R, Gautier L, Nielser HB, Saxild HH, Nielsen C, Brunak S and Knudsen S. A new non-linear normalization method for reducing variability in DNA microarray experiments. Genome Biol. 2002; 3:research0048. 